\title{
High-Resolution Reconstruction for Multidimensional Laplace NMR
}

\author{
Enping Lin, Ville-Veikko Telkki, Xiaoqing Lin, Chengda Huang, Haolin Zhan, Yu Yang,* \\ Yuqing Huang,* and Zhong Chen*
}

Cite This: J. Phys. Chem. Lett. 2021, 12, 5085-5090

Read Online

ACCESS |

WIII Metrics \& More

Article Recommendations

Supporting Information

ABSTRACT: As a perfect complement to conventional NMR that aims for chemical structure elucidation, Laplace NMR constitutes a powerful technique to study spin relaxation and diffusion, revealing information on molecular motions and spin interactions. Different from conventional NMR adopting Fourier transform to deal with the acquired data, Laplace NMR relies on specially designed signal processing and reconstruction algorithms resembling the inverse Laplace transform, and it generally faces severe challenges in cases where high spectral resolution and high spectral dimensionality are required. Herein, based on the tensor technique for high-dimensional problems and the sparsity assumption, we propose a general method

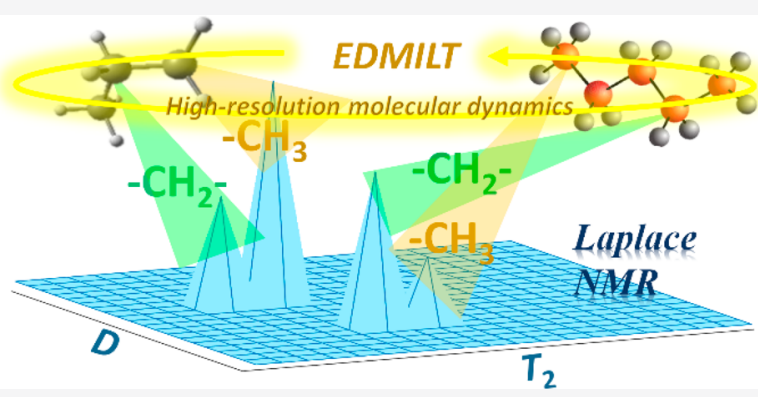
for high-resolution reconstruction of multidimensional Laplace NMR data. We show that the proposed method can reconstruct multidimensional Laplace NMR spectra in a high-resolution manner for exponentially decaying relaxation and diffusion data acquired by commercial NMR instruments. Therefore, it would broaden the scope of multidimensional Laplace NMR applications.

$\mathrm{N}$ MR spectroscopy offers a robust and noninvasive tool for applications in various fields, such as chemistry, biology, and materials science. ${ }^{1}$ As an effective technique revealing atomic level information on chemical shifts and $J$ couplings, conventional NMR experiments (e.g., $1 \mathrm{D} \mathrm{NMR}^{2}$ and $2 \mathrm{D} \mathrm{COSY}^{3}$ ) are widely used in molecular structure determination, conformation elucidation, and composition analysis. ${ }^{4}$ However, relaxation and diffusion parameters related to molecular dynamics and spin interactions ${ }^{5-8}$ are inaccessible by conventional NMR and are generally provided by Laplace NMR experiments. Theoretically, due to the exponentially decaying signals caused by the relaxation and diffusion processes, the Inversion Laplace Transform (ILT) can be adopted to retrieve the distribution of relaxation times or diffusion coefficients. ${ }^{9}$ Compared to conventional NMR experiments, where acquired Free Induction Decay (FID) signals are directly converted to spectral peaks in the frequency domain by the Fourier Transform (FT), Laplace NMR involves more complicated processing algorithms to extract the desired dynamic information on diffusion and relaxation due to the ill-posed nature of the ILT problem. Generally, only approximate results can be obtained from Laplace NMR experimental data based on given constraint conditions.

It is clear that high-resolution Laplace NMR requires efficient data reconstruction algorithms for resolving each spectral peak. Although many ILT reconstruction methods have been proposed, it remains challenging to hit the spot of high-resolution Laplace NMR measurements. Current reconstruction methods employ constraints and regularizations ${ }^{10}$ to deal with the ill-posed ILT. For example, the Maximum
Entropy Method (MaxEnt) ${ }^{11}$ constrains the reconstructed spectra with the maximum entropy for DOSY ${ }^{12}$ processing. The Iterative Thresholding Algorithm for Multi-Exponential Decay $(\mathrm{ITAMeD})^{13}$ uses $\mathrm{L}_{1}$ norm regularization and nonnegativity constraint to reconstruct 1D Laplace NMR spectra. The Low-Rank and Sparsity Inverse Laplace Transform (LRSpILT) ${ }^{14}$ exploits combined $\mathrm{L}_{1}$ norm and nuclear norm regularization as well as non-negativity constraint for $2 \mathrm{D}$ highresolution DOSY ${ }^{12}$ reconstruction. The Constrained Regularization Method for Inverting Data (CONTIN) ${ }^{15}$ adopts the second derivative Tikhonov regularization and non-negativity constraint. Marginal Distributions Constrained Optimization $(\mathrm{MADCO})^{16}$ uses 1D distributions measured with full sampling as constraints in the reconstruction of $2 \mathrm{D}$ distributions. However, because of the efficiency and effectiveness of the reconstruction algorithms which are substantially in demand in the multidimensional ILT reconstruction, these methods still suffer from peak broadening and fail to resolve congested peaks. To address the challenge of congested peaks in ILT reconstructions, we point out that one should focus on the optimization algorithm besides constraints and regularization. In practice, the optimization algorithm decides how to search in the solution space and when to

Received: March 31, 2021

Accepted: May 19, 2021

Published: May 24, 2021 
terminate and outputs the results. Thus, even for the same model, different optimizers yield different efficiencies, which depend on a practical problem. To match the optimization algorithm for the ill-posed ILT problem is of much importance.

In this study, we propose a high-resolution multidimensional Laplace NMR reconstruction method, named Enhanced Discerning Multidimensional Inverse Laplace Transform (EDMILT), based on the sparsity assumption ${ }^{17}$ and the tensor technique. The sparsity assumption states that only a few nonzero points exist in the desired reconstructed results, while the tensor technique promotes the proposed method for $n \mathrm{D}(n \geq 3)$ reconstruction. The EDMILT exploits $\mathrm{L}_{1}$ norm regularization and non-negativity constraint and adopts the Truncated Newton Interior Point Method (TNIPM) ${ }^{18}$ algorithm for iterative optimization. The TNIPM, suitable for the high-resolution multidimensional Laplace NMR reconstruction where a large scale and sparse reconstruction is required, tends to effectively find a series of discrete nonzero points, i.e. a group of sharp spectral peaks, as reconstructed results, thus enhancing adjacent peak separation and achieving high-resolution multidimensional Laplace NMR spectra. This proposed method is a tensor-based multidimensional Laplace NMR reconstruction method considering constraints, regularizations, and algorithm property to deal with the challenge of ill-posed ILT. The EDMILT is robust and effective for $1 \mathrm{D}$ and even $n \mathrm{D}$ Laplace NMR reconstructions, which may serve as a general technical tool for high-dimensional Laplace NMR development.

It is assumed that matrix $\mathbf{K}$ denotes a Laplace kernel whose element $K_{i j}$ is defined as eq 1 for $T_{1}$ relaxation and as eq 2 for $T_{2}$ relaxation or diffusion, $\mathbf{s}$ is the measured relaxation or diffusion data which is normalized preferably before reconstruction, $\mathbf{x}$ is the reconstructed vector of $1 \mathrm{D}$ Laplace NMR spectrum and initialized as a vector whose entries all equal 1 in the TNIPM optimized iteration. The ILT objective function can be formulated as eq 3 .

$$
\begin{aligned}
& K_{i j}=1-\mathrm{e}^{-\alpha_{j} t_{i}} \\
& K_{i j}=\mathrm{e}^{-\alpha_{j} t_{i}} \\
& \arg \min _{\mathbf{x} \geq 0}\|\mathbf{K x}-\mathbf{s}\|_{2}^{2}+\lambda\|\mathbf{x}\|_{1}
\end{aligned}
$$

where $\alpha_{j}$ denotes the $j$ th element of the preset decay constant dictionary $\boldsymbol{\alpha}, t_{i}$ represents the $i$ th entry of the evolution time vector $\mathbf{t}, \mathbf{x} \geq 0$ constrains non-negativity of all elements in $\mathbf{x}$, $\|.\|_{1}$ denotes $L_{1}$ norm, defined as the sum of each element magnitudes. This objective function contains a fidelity term $\|\mathbf{K x}-\mathbf{s}\|_{2}^{2}$, which evaluates the reconstructed result $\mathbf{x}$ by how it contributes to the measurement $\mathbf{s}$, and also a regularization term $\|\mathbf{x}\|_{1}$ for the sparsity constraint on $\mathbf{x}$. After normalizing $\mathbf{s}$, the regularization parameter $\lambda$, which trades off between fidelity and regularization terms, is normally set between 0.001 and 0.1 empirically. The regularization parameter effect on EDMILT reconstructed results is analyzed in the Supporting Information. Due to the sparsity constraint on $\mathbf{x}$, EDMILT is inclined to generate sharp peaks. In this sense, EDMILT keeps a good peak shape for monodisperse samples but not for polydisperse samples whose peaks are intrinsically broad. Actually, EDMILT employs sharp peaks to fit the broad peaks; i.e., EDMILT obtains the average diffusion coefficients (decay constants) of polydisperse components. See the Supporting
Information for more analytical details of EDMILT on the broad peak reconstruction.

For $n \mathrm{D}$ ILT, the reconstruction model is formulated as

$$
\arg \underset{\mathbf{X} \geq 0}{\min }\left\|\mathbf{X}_{\times 1} \mathbf{K}_{1 \times 2} \mathbf{K}_{2 \times 3 \cdot \cdots \times n} \mathbf{K}_{n}-\mathbf{S}\right\|^{2}+\lambda\|\mathbf{X}\|_{1}
$$

where $\mathbf{X}$ denotes the reconstructed tensor of the $n \mathrm{D}$ Laplace NMR spectrum, $\mathbf{K}_{i}$ is the Laplace kernel corresponding to ILT along the $i$ th dimension, $\mathbf{S}$ represents the acquired $n \mathrm{D}$ relaxation or diffusion tensor signal, the symbol $\times_{i}$ represents the $i$-mode product of tensor $\mathbf{X}$ with matrix $\mathbf{K}_{i}, \|$. $\|$ denotes the norm of a tensor defined as the square root of the sum of the absolute squares of its elements. Equation 4 can be reformulated into

$$
\arg \min _{\mathbf{z} \geq 0}\|\mathbf{A z}-\mathbf{y}\|_{2}^{2}+\lambda\|\mathbf{z}\|_{1}
$$

where $\mathbf{A}=\mathbf{K}_{n} \otimes \ldots \otimes \mathbf{K}_{2} \otimes \mathbf{K}_{1}, \otimes$ is the Kronecker product, and $\mathbf{z}$ and $\mathbf{y}$ denote column vectorization of $\mathbf{X}$ and $\mathbf{S}$, respectively. We point out that, as analyzed in the Supporting Information, the higher dimensional EDMILT performs better than the lower dimensional one in spectral resolution but at the cost of computational complexity of eq 5. Fortunately, due to the singularity of $\mathbf{A}$, the computational complexity of eq 5 can be significantly reduced by the design of a compressed model detailed in the Supporting Information and then solved efficiently by the TNIPM optimization iterations. TNIPM employs a logarithm barrier function to build a "wall" between negative and positive real number fields for the non-negative constraint of $\mathbf{z}$ during iterations. In each iteration, the TNIPM exploits Newton's method to decrease the objective function (eq 5), which provides a rapid convergence to the optimal point, ${ }^{19}$ thus preventing the optimized variable from oscillating in the neighborhood of the optimal point in an ill-posed ILT problem. We detail the advantage of convergence performance of EDMILT over ITAMed in the Supporting Information. Benefiting from the rapid convergence of the TNIPM optimization algorithm, the EDMILT can reconstruct multidimensional Laplace NMR in a high-resolution manner, which is the most important significance of this work. For a detailed mathematical derivation of the TNIPM iteration for solving the sparse multidimensional ILT model, one can refer to the Supporting Information. All computations in this work are implemented on the MATLAB 2016b platform installed in a desktop with an i7-7700 $3.60 \mathrm{GHz}$ CPU, 16G memory 64 bit Windows 10 operation system.

The resolution of the EDMILT result relies on the Signal Noise Ratio (SNR) and the number of dimensions. For data with a better SNR or higher dimensions, the peak discerning ability of EDMILT will be enhanced. Since the size of A increases with the number of dimensions, higher dimensional reconstruction needs more computing time and memory. We present more detailed analysis of EDMILT, including related mathematical derivation, peak discerning analysis, reconstruction time, and access to MATLAB codes of EDMILT, whose iterative optimized part is from Kim et al.'s code ${ }^{18}$ with some simplifications for the specific ILT inverse problem, in the Supporting Information.

To evaluate the performance of the EDMILT, we perform reconstruction tests on three kinds of Laplace NMR experimental data. First, 2D Diffusion-Ordered Spectroscopy (DOSY) data ${ }^{12,20}$ of a mixture sample containing sucrose, lysine, threonine, butanol, ethanol, and methanol (named as 
M6 for convenience in following) is adopted for the basic 1D Laplace reconstruction. 2D DOSY, containing chemical shift and diffusion coefficient information along two orthogonal dimensions, constitutes a standard tool for chemical component separation and mixture analysis. 2D DOSY belongs to 1D Laplace experiments since it only contains one dimension of relaxation or diffusion. The parameters of this experimental data are detailed in ref 21.

Second, to show the excellent peak discerning performance of EDMILT for 2D Laplace reconstruction, we test it on 2D Laplace NMR data acquired from diffusion and $T_{2}$ relaxation $\left(D-T_{2}\right)$ correlation experiments on a mixture of $1.36 \mathrm{M}$ hexane $\left(\mathrm{C}_{6} \mathrm{H}_{14}\right)$ and $0.79 \mathrm{M}$ pentadecane $\left(\mathrm{C}_{15} \mathrm{H}_{32}\right)$. The $D-T_{2}$ correlation experiment was obtained using gradient-based ultrafast Laplace methods (UF-LNMR, see ref 22) for fast and accurate component analysis.

Finally, we perform a 3D Laplace reconstruction test on a homemade $D-T_{2}-T_{1}$ data of a mixture of $0.96 \mathrm{M}$ cyclohexane $\left(\mathrm{C}_{6} \mathrm{H}_{12}\right)$ and $1.02 \mathrm{M}$ tetradecane $\left(\mathrm{C}_{15} \mathrm{H}_{32}\right)$ (detailed experimental parameters are given in Supporting Information) to show the ability of the EDMILT for reconstruction on highresolution multidimensional Laplace NMR spectra. To further show the performance of the EDMILT on high-resolution multidimensional Laplace NMR reconstruction, we show more 3D Laplace simulated reconstructed results in the Supporting Information.

First, we compare EDMILT with ITAMeD, ${ }^{13}$ a $1 \mathrm{D}$ diffusion reconstruction method, and LRSpILT, ${ }^{14}$ a $2 \mathrm{D}$ DOSY reconstruction method, for $2 \mathrm{D}$ DOSY spectrum reconstruction of the M6 mixture. It is noteworthy that many other processing methods $^{23-28}$ have been proposed since the invention of DOSY and that Nilsson has integrated many DOSY processing data into a free software named DOSYToolbox. ${ }^{29}$ Here we select the excellent and relatively recent ITAMeD and LRSpILT for comparison. From the reconstructed DOSY spectra (Figure 1), it is clear that the results obtained by ITAMeD and LRSpILT contain artifacts as marked by arrows, while the DOSY spectrum by the EDMILT shows all signal peaks and presents only one shifted peak. For a detailed analysis of peak width along the diffusion dimension obtained by these three methods, we project all peaks of normalized reconstructed spectra into the diffusion dimension, as shown in Figure S4. We select spectral points with significant intensity values that are above a threshold of 0.01 as signal peaks to calculate Mean Peak Widths (MPWs), which are equal to $0.520,1.125$, and $0.215\left(10^{-10} \mathrm{~m}^{2} \mathrm{~s}^{-1}\right)$ for ITAMeD (Figure 1a), LRSpILT (Figure 1b), and EDMILT (Figure 1c), respectively. Since smaller MPW corresponds to sharper peak and higher spectral resolution, we can infer from MPWs that the EDMILT possesses higher resolution than other reconstruction methods do.

To show the performance of the EDMILT on separating severely congested peaks, we mark five groups of overlapping peaks in Figure 1 with yellow dash lines in numerical order. Ideally, overlapping peaks of groups $1-5$ lie in sucrose and lysine components, sucrose and ethanol components, threonine and butanol components, lysine and butanol components, and threonine and butanol components, respectively. Because of the low resolution of the diffusion dimension, overlapping peaks of groups $1,3,4$, and 5 in the DOSY spectrum by the ITAMeD are merged into broad peaks along the diffusion dimension marked with green arrows in

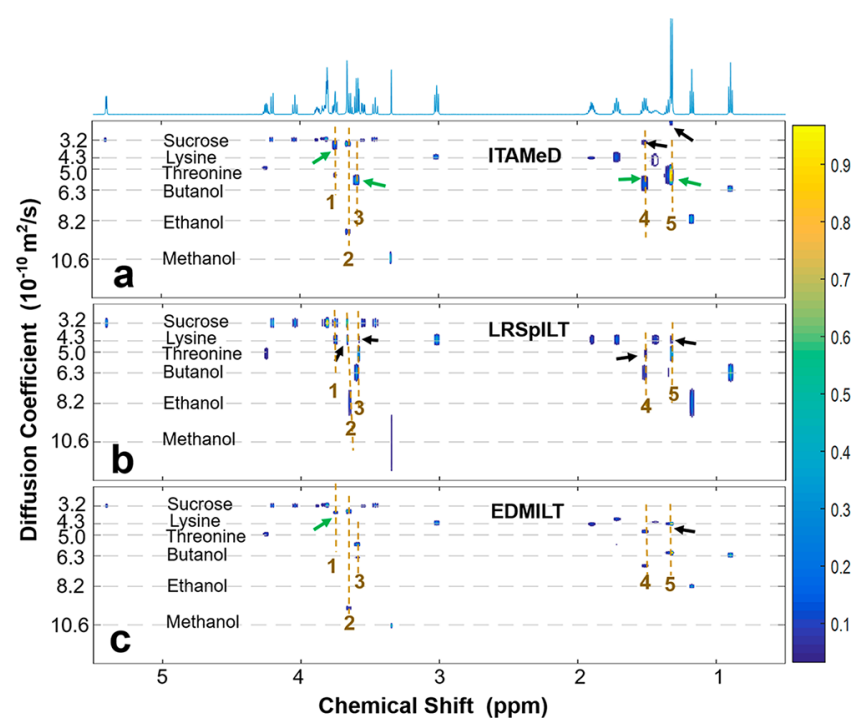

Figure 1. Reconstructed 2D DOSY NMR spectra for M6, a mixture containing sucrose, lysine, threonine, butanol, ethanol, and methanol, by (a) ITAMeD, (b) LRSpILT, and (c) EDMILT. All the three method are terminated when the relative difference of neighbor iterative outputs is less than $10^{-5}$. ITAMeD and EDMILT use one compromise regularization parameter 0.03 for reconstruction. The overlapping peaks are marked by yellow dashed lines and order numbers. Artifacts or shifted peaks are marked by black arrows, merged peaks are marked by green arrows, and diffusion coefficient values of components are marked by gray dashed lines.

Figure $1 \mathrm{a}$ and present two undesired artifacts marked with black arrows.

As for the LRSpILT, overlapping peaks of groups 2-5 appear artifacts along the diffusion dimension, caused by adjacent component peak broadenings (Figure 1b). For example, in the region of group 2, which should contain only sucrose and ethanol components, an artifact appears as the lysine component. In the region of group 4, a peak marked with a black arrow is also an artifact, which should hold the diffusion coefficient of lysine but instead is located in the threonine position.

As for the EDMILT, groups 2-4 of overlapping peaks are clearly discerned along the diffusion dimension (Figure 1c). Group 1 merges into a compromise peak, and group 5 has a peak shift. Although there exist diffusion coefficient differences among the same mixture component in the DOSY spectrum by the EDMILT, e.g., one peak around $3.7 \mathrm{ppm}$ from the sucrose slightly deviated from the ideal component reference lines along the diffusion dimension, it would not influence the component separations and assignments severely. It is noteworthy that peaks of greater diffusion coefficients are much broader in the LRSpILT reconstructed spectra. The reason is that, in the PFG diffusion NMR experiment, components with greater diffusion coefficients generate signals with stronger attenuation, which are more sensitive to the nonuniform pulse gradients than those with smaller diffusion coefficients. $^{30}$ However, in the ITAMeD and EDMILT reconstructed spectra, greater diffusion coefficient components still present narrow peaks, benefiting from the sparsity regularization. In this sense, ITAMeD and EDMILT can diminish the effect of nonuniform pulse gradients.

Furthermore, we compare EDMILT with two 2D ILT methods. The first one is CONTIN, ${ }^{15}$ in which the $\mathrm{L}_{2}$ norm of 
the second derivative is used as a regularizer and nonnegativeness is constrained for dealing with the 2D ILT inverse problem. The second one, ${ }^{31}$ which is called "common 2D ILT" in the following, was proposed based on refs 32 and 33 and has been used in several famous works ${ }^{7,22,31,34}$ for reconstructing 2D Laplace NMR spectra. Figure 2 shows reconstructed

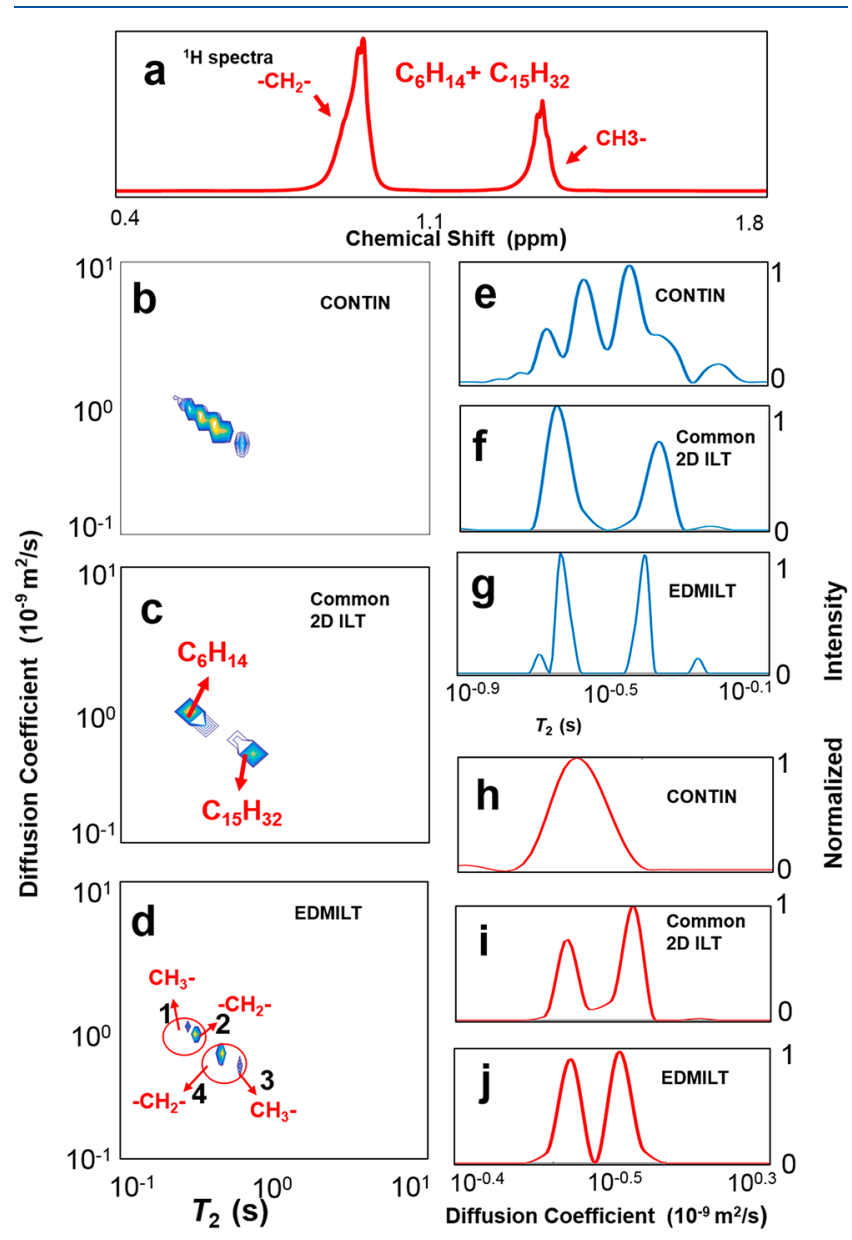

Figure 2. ${ }^{1} \mathrm{H}$ NMR spectra (a) and reconstructed $D-T_{2}$ correlation spectra for a mixture of $1.36 \mathrm{M}$ hexane $\left(\mathrm{C}_{6} \mathrm{H}_{14}\right)$ and $0.79 \mathrm{M}$ pentadecane $\left(\mathrm{C}_{15} \mathrm{H}_{32}\right)$ dissolved in $\mathrm{CCl}_{4}$ by CONTIN (b), the common 2D ILT method (c), and the EDMILT (d). Spectra (b)-(d) are projected along the $T_{2}$ relaxation dimension shown in $(\mathrm{e})-(\mathrm{g})$ and along the diffusion dimension shown in $(\mathrm{h})-(\mathrm{j})$.

spectra by three methods from $D-T_{2}$ correlation data performed on a mixture of $1.36 \mathrm{M}$ hexane $\left(\mathrm{C}_{6} \mathrm{H}_{14}\right)$ and 0.79 $M$ pentadecane $\left(\mathrm{C}_{15} \mathrm{H}_{32}\right)$. It can be noticed that hexane and pentadecane share similar chemical shift sites and yield completely overlapped peaks in the standard $1 \mathrm{D}{ }^{1} \mathrm{H}$ NMR spectrum (Figure 2a), making component separation by conventional DOSY challenging. The $D-T_{2}$ experiments effectively address this challenge by adding $T_{2}$ evolution along the other dimension. Figure 2 shows compared $D-T_{2}$ correlation spectra by CONTIN, the common $2 \mathrm{D}$ ILT method, and the EDMILT.

In the CONTIN reconstructed result (Figure $2 \mathrm{~b}$ ), peaks are congested due to the smoothness effect, which can be seen in both the $T_{2}$ relaxation and the diffusion projected spectra. In Figure $2 \mathrm{e}$, four peaks indicating methyl $\left(-\mathrm{CH}_{3}\right)$ and methylene $\left(-\mathrm{CH}_{2}\right)$ components on the $T_{2}$ relaxation dimension partially overlap in the base. In Figure $2 \mathrm{~h}$, only one broad peak appears, implying failure to discern molecular components. The common $2 \mathrm{D}$ ILT method can separate hexane and pentadecane components along the diffusion dimension but fails to further discern methyl $\left(-\mathrm{CH}_{3}\right)$ and methylene $\left(-\mathrm{CH}_{2}\right)$ components on the $T_{2}$ relaxation dimension. Figure $2 \mathrm{f}$ and $\mathrm{i}$ shows that two peaks exist in both the $T_{2}$ relaxation spectrum and the diffusion spectrum projected from the $D-T_{2}$ spectrum reconstructed by the common 2D ILT method (Figure 2c). By contrast, the EDMILT presents four peaks in the reconstructed highresolution $D-T_{2}$ spectrum (Figure $2 \mathrm{~d}$ ). According to the projected spectra in Figure $2 \mathrm{~g}$ and $\mathrm{j}$, we can see four peaks in the $T_{2}$ relaxation projected spectrum and two peaks in the diffusion projected spectrum. Although these peaks are not completely aligned along the diffusion dimension, it still distinguishes the two components. The EDMILT differentiates two peaks along the diffusion dimension, standing for two components of hexane and pentadecane, and discerns four peaks along the $T_{2}$ dimension, representing methyl $\left(-\mathrm{CH}_{3}\right)$ and methylene $\left(-\mathrm{CH}_{2}\right)$ in these two components.

Compared to $1 \mathrm{D}$ and 2D Laplace NMR, higher dimensional Laplace NMR can provide superior signal dispersion and give more extensive dynamic information on relaxation and diffusion. The applicability of existing ILT methods on 3D Laplace NMR is generally limited by processing time consumption and reconstructed spectral resolution. Here, the EDMILT is applied to $D-T_{2}-T_{1}$ experimental data of a mixture of cyclohexane $\left(\mathrm{C}_{6} \mathrm{H}_{12}\right)$ and tetradecane $\left(\mathrm{C}_{15} \mathrm{H}_{32}\right)$ to recover the desired 3D Laplace NMR spectra. For convenient analysis, we label four proton groups as $1-4$ in Figure 3. As shown in

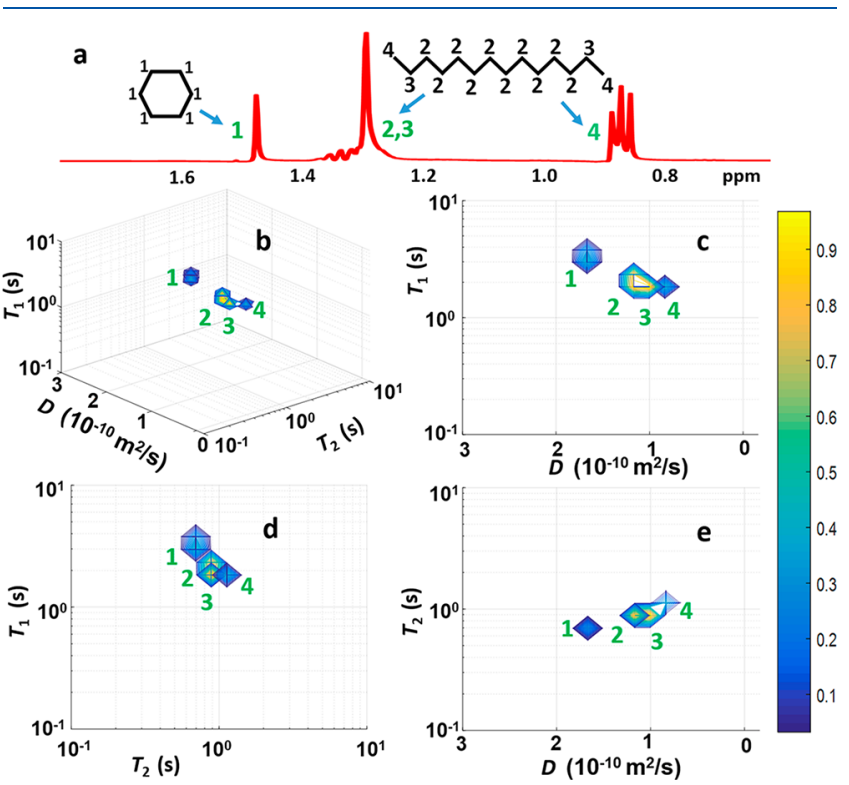

Figure 3. ${ }^{1} \mathrm{H}$ NMR spectrum (a) and reconstructed 3D $D-T_{2}-T_{1}$ correlation spectrum for the mixture of cyclohexane and tetradecane reconstructed by the EDMILT (b) and its $2 \mathrm{D} D-T_{1}$ (c), $T_{2}-T_{1}$ (d), and $D-T_{2}$ (e) projected spectra. All spectra have been normalized.

Figure 3a, peaks of groups 2 and 3 overlap severely in standard 1D ${ }^{1} \mathrm{H}$ NMR spectra. In the $D-T_{2}-T_{1}$ spectrum by the EDMILT (Figure $3 \mathrm{~b}$ ), four peaks are presented conspicuously for the following component identification and dynamic analysis on relaxation and diffusion. For further analysis of the reconstruction result, $2 \mathrm{D}$ projected spectra of $D-T_{1}, T_{2}-T_{1}$, and $D-T_{2}$ are shown in Figure $3 c-e$, respectively. From Figure 
$3 c$ and e, it can be seen that peak groups 2-4 are close along the diffusion $(D)$ dimension, which indicates they belong to the identical tetradecane component, while peak group 1 belongs to cyclohexane. In Figure $3 c-e$, peaks 3 and 4 overlap along the $T_{1}$ dimension, and peaks 2 and 3 overlap along the $T_{2}$ dimension because of the similar chemical environment. However, all these relaxation and diffusion information can be clearly separated and identified in the resulting 3D Laplace spectrum benefiting from its ability of signal dispersion. In addition, correlation $2 \mathrm{D}$ spectra between relaxation and diffusion parameters, i.e., $D-T_{1}, T_{2}-T_{1}$, and $D-T_{2}$, can also be used for practical analysis, such as component phase exchange and molecule interactions, which are obtained simultaneously by the 3D Laplace NMR. Therefore, benefiting from the satisfactory performance on 3D Laplace NMR reconstruction, the EDMILT may extend the scope of Laplace NMR to higher dimensional applications.

The spectral resolution is the crucial factor determining the usability of acquired NMR spectra. Compared to conventional NMR experiments in which spectral resolution is generally determined by given experimental parameters, such as acquisition points and spectral width, Laplace NMR experiments rely on data processing and reconstruction method to yield high-resolution information, in addition to the experimental parameters. The EDMILT reconstruction implementation on aforementioned complex mixtures suggests that the EDMILT presents a useful tool for multidimensional Laplace NMR experiments to achieve high-resolution spectral reconstruction and offers chemical resolution not achievable in conventional NMR spectra, and not even in traditional Laplace NMR, thus benefiting molecular dynamic analysis in practical applications. Laplace NMR focuses on dynamic information on relaxation and diffusion and avoids molecular structure information on chemical shifts and $J$ couplings that are highly dependent on static magnetic fields. Therefore, the EDMILT aiming for high-resolution reconstruction is also suitable for low-field Laplace NMR applications, e.g., petroleum exploration, portable food detection, or mobile NMR measurements. ${ }^{35}$ However, EDMILT might not result in the intrinsic peak profile for polydisperse samples. In other words, EDMILT might not be applicable for obtaining the ideal diffusion coefficient distribution ranges of polydisperse components. Actually, EDMILT employs sharp peaks to fit the broad peaks and obtains the average diffusion coefficients (decay constants) of polydisperse components.

In conclusion, we propose a general NMR reconstruction method for resolving multidimensional ILT problems and achieve high-resolution Laplace NMR spectra based on tensor techniques and sparsity assumption. This method can be applied to dynamic related NMR experiments and serve as a tool for the future development of multidimensional Laplace NMR spectroscopy. The experiments prove that this proposed method can effectively recover dynamic information related to diffusion and relaxation behaviors in a high-resolution manner compared to the state-of-the-art ILT methods. Thus, it may promote the development of multidimensional Laplace NMR as an essential branch of NMR spectroscopy and shows a promising prospect for diverse applications of Laplace NMR.

\section{ASSOCIATED CONTENT}

\section{s) Supporting Information}

The Supporting Information is available free of charge at https://pubs.acs.org/doi/10.1021/acs.jpclett.1c01022.
Compressed model for the EDMILT; TNIPM algorithm detail for the EDMILT; detailed experimental parameters of $D-T_{2}-T_{1}$ data of a mixture of $0.96 \mathrm{M}$ cyclohexane $\left(\mathrm{C}_{6} \mathrm{H}_{12}\right)$ and $1.02 \mathrm{M}$ tetradecane $\left(\mathrm{C}_{15} \mathrm{H}_{32}\right)$; convergence efficacy comparison of EDMILT and ITAMeD; resolution analysis for DOSY NMR of M6; performance analysis of the EDMILT; 2D and 3D simulated Laplace spectra reconstructed by the EDMILT; analytical details of EDMILT on polydisperse samples; regularization parameter effect on the EDMILT (PDF)

\section{AUTHOR INFORMATION}

\section{Corresponding Authors}

Zhong Chen - State Key Laboratory of Physical Chemistry of Solid Surfaces, Xiamen University, Xiamen, Fujian 361005, China; (1) orcid.org/0000-0002-1473-2224;

Email: chenz@xmu.edu.cn

Yuqing Huang - State Key Laboratory of Physical Chemistry of Solid Surfaces, Xiamen University, Xiamen, Fujian 361005, China; ㅇo orcid.org/0000-0003-0606-1165; Email: yqhuangw@xmu.edu.cn

Yu Yang - State Key Laboratory of Physical Chemistry of Solid Surfaces, Xiamen University, Xiamen, Fujian 361005, China; orcid.org/0000-0002-5514-455X;

Email: yuyang15@xmu.edu.cn

\section{Authors}

Enping Lin - State Key Laboratory of Physical Chemistry of Solid Surfaces, Xiamen University, Xiamen, Fujian 361005, China

Ville-Veikko Telkki - NMR Research Unit, University of Oulu, Oulu FIN-90014, Finland; (1) orcid.org/0000-00030846-6852

Xiaoqing Lin - State Key Laboratory of Physical Chemistry of Solid Surfaces, Xiamen University, Xiamen, Fujian 361005, China

Chengda Huang - State Key Laboratory of Physical Chemistry of Solid Surfaces, Xiamen University, Xiamen, Fujian 361005, China

Haolin Zhan - State Key Laboratory of Physical Chemistry of Solid Surfaces, Xiamen University, Xiamen, Fujian 361005, China; (1) orcid.org/0000-0001-8202-5639

Complete contact information is available at:

https://pubs.acs.org/10.1021/acs.jpclett.1c01022

\section{Notes}

The authors declare no competing financial interest.

\section{ACKNOWLEDGMENTS}

This work was supported by National Natural Science Foundation of China (U1805261, 11761141010, 22073078, and 61601386). E.L. thanks Dr. Bin Yuan for providing DOSY experimental data of the sample M6. V.-V.T. acknowledges the financial support of the European Research Council (ERC) under Horizon 2020 (H2020/2018-2022/ERC Grant Agreement No. 772110) and Kvantum Institute (University of Oulu).

\section{REFERENCES}

(1) Larive, C. K.; Barding, G. A.; Dinges, M. M. NMR spectroscopy for metabolomics and metabolic profiling. Anal. Chem. 2015, 87 (1), 133-146. 
(2) Englert, G.; Bjørnland, T.; Liaaen-Jensen, S. 1D and 2D NMR study of some allenic carotenoids of the fucoxanthin series. Magn. Reson. Chem. 1990, 28 (6), 519-528.

(3) Aue, W. P.; Bartholdi, E.; Ernst, R. R. Two-dimensional spectroscopy. Application to nuclear magnetic resonance. J. Chem. Phys. 1976, 64 (5), 2229-2246.

(4) Venkatesh, A.; Lund, A.; Rochlitz, L.; Jabbour, R.; Gordon, C. P.; Menzildjian, G.; Viger-Gravel, J.; Berruyer, P.; Gajan, D.; Copéret, C.; Lesage, A.; Rossini, A. J. The structure of molecular and surface platinum sites determined by DNP-SENS and fast MAS 195Pt solidstate NMR spectroscopy. J. Am. Chem. Soc. 2020, 142 (44), 1893618945 .

(5) Telkki, V. V. Hyperpolarized Laplace NMR. Magn. Reson. Chem. 2018, 56, 619-632.

(6) King, J. N.; Lee, V. J.; Ahola, S.; Telkki, V. V.; Meldrum, T. Ultrafast multidimensional Laplace NMR using a single-sided magnet. Angew. Chem., Int. Ed. 2016, 55 (16), 5040-5043.

(7) Mankinen, O.; Zhivonitko, V. V.; Selent, A.; Mailhiot, S.; Komulainen, S.; Prisle, N. L.; Ahola, S.; Telkki, V. V. Ultrafast diffusion exchange nuclear magnetic resonance. Nat. Commun. 2020, 11 (1), 3251.

(8) Busse, F.; Rehorn, C.; Küppers, M.; Ruiz, N.; Stege, H.; Blümich, B. NMR relaxometry of oil paint binders. Magn. Reson. Chem. 2020, 58 (9), 830-839.

(9) Lee, J. H.; Labadie, C.; Springer, C. S.; Harbison, G. S. Twodimensional inverse Laplace transform NMR: altered relaxation times allow detection of exchange correlation. J. Am. Chem. Soc. 1993, 115 (17), 7761.

(10) Ames, K. A.; Clark, G. W.; Epperson, J. F.; Oppenheimer, S. F. A comparison of regularizations for an ill-posed problem. Math. Comput. 1998, 67 (224), 1451-1471.

(11) Delsuc, M. A.; Malliavin, T. E. Maximum entropy processing of DOSY NMR spectra. Anal. Chem. 1998, 70 (10), 2146-2148.

(12) Morris, K. F.; Johnson, C. S. Diffusion-ordered two-dimensional nuclear magnetic resonance spectroscopy. J. Am. Chem. Soc. 1992, 114 (8), 3139-3141.

(13) Urbańczyk, M.; Bernin, D.; KozMiński, W.; Kazimierczuk, K. Iterative thresholding algorithm for multiexponential decay applied to PGSE NMR data. Anal. Chem. 2013, 85 (3), 1828-1833.

(14) Lin, E.; Yang, Y.; Huang, Y.; Chen, Z. High-resolution reconstruction for diffusion-ordered NMR spectroscopy. Anal. Chem. 2020, 92 (1), 634-639.

(15) Provencher, S. W. CONTIN: A general purpose constrained regularization program for inverting noisy linear algebraic and integral equations. Comput. Phys. Commun. 1982, 27 (3), 229-242.

(16) Benjamini, D.; Basser, P. J. Use of marginal distributions constrained optimization (MADCO) for accelerated 2D MRI relaxometry and diffusometry. J. Magn. Reson. 2016, 271, 40-45.

(17) Selesnick, I. Sparse regularization via convex analysis. IEEE Trans. Signal Process. 2017, 65 (17), 4481-4494.

(18) Kim, S.-J.; Koh, K.; Lustig, M.; Boyd, S.; Gorinevsky, D. An interior-point method for large-scale L1-regularized least squares. IEEE J. Sel. Top. Signal Process. 2007, 1 (4), 606-617.

(19) Boyd, S.; Vandenberghe, L. Convex optimization; Cambridge University Press, 2013.

(20) Foroozandeh, M.; Castañar, L.; Martins, L. G.; Sinnaeve, D.; Poggetto, G. D.; Tormena, C. F.; Adams, R. W.; Morris, G. A.; Nilsson, M. Ultrahigh-resolution diffusion-ordered spectroscopy. Angew. Chem., Int. Ed. 2016, 55 (50), 15579-15582.

(21) Yuan, B.; Ding, Y.; Kamal, G. M.; Shao, L.; Zhou, Z.; Jiang, B.; Sun, P.; Zhang, X.; Liu, M. Reconstructing diffusion ordered NMR spectroscopy by simultaneous inversion of Laplace transform. J. Magn. Reson. 2017, 278, 1-7.

(22) Ahola, S.; Zhivonitko, V. V.; Mankinen, O.; Zhang, G.; Kantola, A. M.; Chen, H. Y.; Hilty, C.; Koptyug, I. V.; Telkki, V. V. Ultrafast multidimensional Laplace NMR for a rapid and sensitive chemical analysis. Nat. Commun. 2015, 6, 8363.
(23) Nilsson, M.; Connell, M. A.; Davis, A. L.; Morris, G. A. Biexponential fitting of diffusion-ordered NMR data: practicalities and limitations. Anal. Chem. 2006, 78 (9), 3040-3045.

(24) Nilsson, M.; Morris, G. A. Speedy component resolution: an improved tool for processing diffusion-ordered spectroscopy data. Anal. Chem. 2008, 80 (10), 3777-3782.

(25) Provencher, S. W. An eigenfunction expansion method for the analysis of exponential decay curves. J. Chem. Phys. 1976, 64 (7), $2772-2777$

(26) Stilbs, P.; Paulsen, K.; Griffiths, P. C. Global least-squares analysis of large, correlated spectral data sets: application to component-resolved FT-PGSE NMR Spectroscopy. J. Phys. Chem. 1996, 100 (20), 8180-8189.

(27) Windig, W.; Antalek, B. Direct exponential curve resolution algorithm (DECRA): A novel application of the generalized rank annihilation method for a single spectral mixture data set with exponentially decaying contribution profiles. Chemom. Intell. Lab. Syst. 1997, 37 (2), 241-254.

(28) Van Gorkom, L. C. M.; Hancewicz, T. M. Analysis of DOSY and GPC-NMR experiments on polymers by multivariate curve resolution. J. Magn. Reson. 1998, 130 (1), 125-30.

(29) Nilsson, M. The DOSY Toolbox: A new tool for processing PFG NMR diffusion data. J. Magn. Reson. 2009, 200 (2), 296-302.

(30) Morris, G. A. Diffusion-ordered spectroscopy. eMagRes 2009, DOI: 10.1002/9780470034590.emrstm0119.pub2.

(31) Godefroy, S.; Callaghan, P. T. 2D relaxation/diffusion correlations in porous media. Magn. Reson. Imaging 2003, 21 (34), 381-383.

(32) Song, Y. Q.; Venkataramanan, L.; Hürlimann, M. D.; Flaum, M.; Frulla, P.; Straley, C. T(1)-T(2) correlation spectra obtained using a fast two-dimensional Laplace inversion. J. Magn. Reson. 2002, 154 (2), 261-268.

(33) Venkataramanan, L.; Song, Y. Q.; Hurlimann, M. D. Solving Fredholm integrals of the first kind with tensor product structure in 2 and 2.5 dimensions. IEEE Trans. Signal Process 2002, 50 (5), 10171026.

(34) Javed, M. A. A.; Ahola, S.; HåKansson, P.; Mankinen, O.; Aslam, M. K.; Filippov, A.; Shah, F. U.; Glavatskih, S.; Antzutkin, O. N.; Telkki, V. V. Structure and dynamics elucidation of ionic liquids using multidimensional Laplace NMR. Chem. Commun. 2017, 53, 11056-11059.

(35) Casanova, F.; Perlo, J.; Blümich, B. Single-sided NMR; Springer: Berlin Heidelberg, 2011. 